\title{
Tratamento Cirúrgico de Hálux Valgo Através de Técnica Minimamente Invasiva - Relato de Caso
}

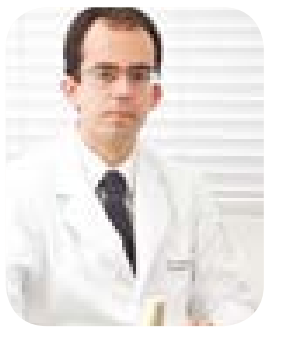

\author{
Fernando Delmonte Moreira ${ }^{1}$, Jorge Eduardo S. Jambeiro', \\ Antero Tavares Neto ${ }^{1}$, Walter Silva de A. Júnior ${ }^{1}$
}

\section{INTRODUÇÃO}

O hálux valgo (HV) é uma deformidade comum entre as patologias do pé e tornozelo, com etiologia multifatorial. A primeira publicação sobre o assunto foi escrita por Carl Hueter, em 1870. Clinicamente, o HV está relacionado às queixas do hálux, como dificuldade de vestir calçados, dores, deformidades e alterações nos dedos laterais. A avaliação inicial depende de um exame físico estático e dinâmico adequado de todo o membro e estudo radiográfico ${ }^{3,4}$.

Trata-se de uma deformidade irreversível do antepé, caracterizada por um desvio lateral (valgo) do hálux e medial (aduto) do $1^{\circ}$ metatarsal (MTT), algumas vezes associada à pronação do $1^{\circ}$ dedo ${ }^{3,6}$.

A proeminência medial observada na articulação metatarsofalangiana (bunion) está presente em alguns casos, sendo causada por um aumento crônico das estruturas mediais e pela lateralização do $1^{\circ}$ metatarsal. Quanto à epidemiologia, sabe-se que a hereditariedade é considerada o fator predisponente mais relevante, com alguns estudos mostrando uma tendência familiar em $68 \%$ dos casos. Entre os pacientes com HV, $80 \%$ queixam-se de restrição ao uso de algum tipo de calçado, $70 \%$ apresentam dor na eminência medial, $60 \%$ procuram atendimento por questões estéticas e $40 \%$ apresentam comprometimento do $2^{\circ}$ dedo, como garra e metatarsalgia ${ }^{4,5,6}$.

A patologia tem uma incidência maior na população idosa, com acometimento de 15 mulheres para cada homem. Segundo Gould et al., 1 a cada 45 indivíduos a partir dos 50 anos apresenta HV. Diversos fatores intrínsecos e extrínsecos estão relacionados à gênese do HV. Entre os intrínsecos, podemos citar: pé plano, hipermobilidade do $1^{\circ}$ raio, conformação da falange proximal, do $1^{\circ} \mathrm{MTT}$ e da MTT-FL e características da cápsula medial. $\mathrm{O}$ fator extrínseco mais importante é o uso de calçados inadequados, com câmara anterior estreita, não permitindo a acomodação do antepé, estimulando o valgismo da $1^{\text {a }}$ MTT-F ${ }^{3,4,5,6}$.
O diagnóstico é clínico e radiográfico. Avalia-se o alinhamento do retropé (valgo, neutro ou varo), as características do arco plantar longitudinal (plano, normal ou cavo), a postura do antepé (aduto/abduzido, supinado/pronado) e os dedos isoladamente (deformidades em varo, valgo, extensão e flexão). Manobras de redução, como a descrita por McBride, também são importantes ${ }^{1,3,4,5,6}$. Coughlin, em 1996, classificou a gravidade da deformidade do HV, como mostra a Tabela I, através da mensuração dos ângulos metatarsofalangeano e intermetatarsal, e deslocamento do sesamoide.

Tabela 1 - Classificação de Coughlin para gravidade da deformidade do hálux valgo

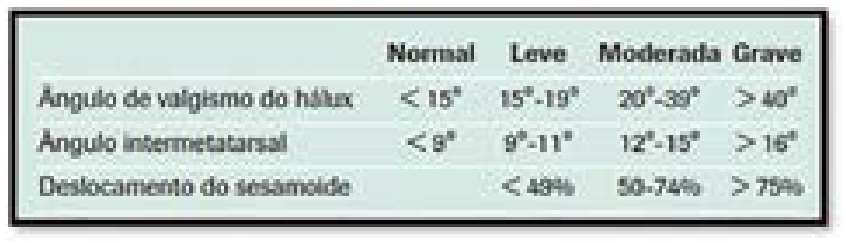

O objetivo do tratamento é aliviar a dor, melhorando a deambulação. O tratamento do HV inicialmente é conservador, com analgésicos, anti-inflamatórios, mudança das atividades e, principalmente, adequação do calçado. Deve-se orientar o paciente a utilizar calçados com a câmara anterior ampla o suficiente para acomodar o antepé e com um solado firme, distribuindo de forma homogênea a pressão na face plantar do pé durante todas as fases da marcha, e salto de até 4 $\mathrm{cm}$. É importante deixar claro para o paciente que esse tratamento visa à melhora da dor e da função, sem corrigir a deformidade ${ }^{3,4,6}$.

Se após o tratamento conservador correto não houver melhora da dor e da função, o tratamento cirúrgico está indicado. Mais de 140 procedimentos foram desenvolvidos para tratar o HV. Para escolher o mais adequado para cada paciente, é necessário levar em conta o grau 
de função e atividade, doenças associadas, características mecânicas da patologia em si e a gravidade da deformidade. Para fins didáticos, pode-se dividir as cirurgias de correção de HV em: liberação de partes moles distais, procedimentos ósseos distais, procedimentos ósseos proximais, osteotomias e artrodeses ${ }^{3,4,5,6}$.

No caso exposto optou-se por utilizar a cirurgia percutânea, ou cirurgia minimamente invasiva (MIS), como ficou conhecida. Esta modalidade cirúrgica é realizada através de incisões puntiformes sem exposição direta do sítio cirúrgico, com auxílio de radioscopia e mínima agressão aos tecidos adjacentes. Trata-se de um modo diferente de realizar procedimentos convencionais, com menor agressão de partes moles, exposição óssea, dor pós-operatória e tempo cirúrgico, em que se utiliza um motor elétrico de 5 a $10 \mathrm{~N}$ de força, com rotação de 7000 RPM; brocas específicas Shannon de corte e Wedge cônica e cilíndrica de tamanhos diferentes, dependendo do caso; um bisturi e instrumental específicos e parafusos de fixação, eventualmente $2,9.10$.

Como as abordagens são feitas de forma percutânea, sem visualização direta, é necessário o profundo conhecimento anatômico das estruturas envolvidas, fato que demanda grande curva de aprendizado, a fim de evitar lesões iatrogênicas ${ }^{2,7,8,9,10}$.

Trata-se de uma técnica desenvolvida há 65 anos, mas que teve destaque apenas a partir de 1985, nos EUA, com Stephen Isham, na década de 1990, com Mariano Prado e Pau Golano, na Europa, e em 2002, na França, com o grupo GRECMIP (Groupe de Recherche et d'Etude en Chirurgie Mini-Invasive du Pied ) 7,8.9,10.

\section{CASO CLÍNICO}

Paciente RCCC, 54 anos, sexo feminino, procedente de Salvador, acompanhada pelo grupo de pé e tor- nozelo do Hospital Santa Izabel no Ambulatório Silva Lima, portadora de hálux valgobilateral, grave à direita (ângulo IM=16, ângulo $M F=34^{\circ}$ ). (Figura 1)

O exame físico mostrava um quadro de hálux valgo com bunion volumoso característico, associado à dor, dificuldade de utilizar calçados fechados e refratário ao tratamento conservador por 6 meses. Como comorbidade a paciente apresentava insuficiência venosa periférica, não sendo contraindicação ao tratamento cirúrgico proposto. (Figura 2).
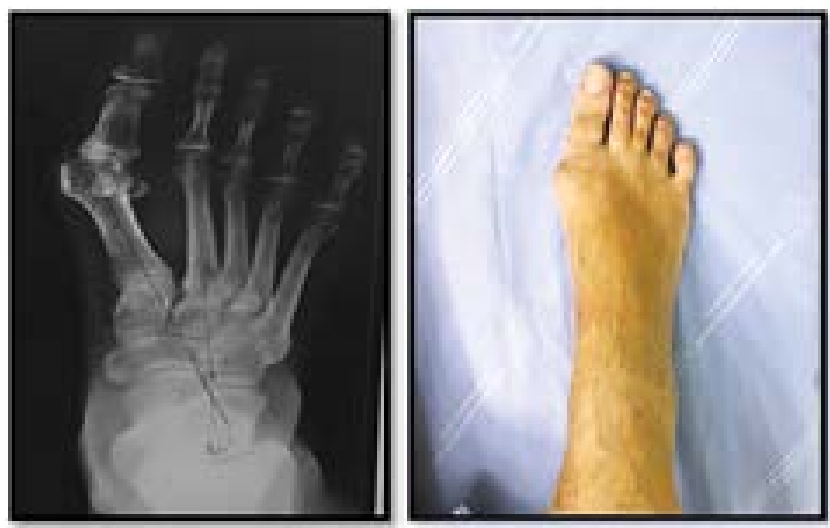

Figuras 1 e 2

Foi utilizado o questionário AOFAS no pré-operatório. Essa escala pontua oito fatores, de zero a 100 pontos, relacionados ao hálux valgo, tais como: dor, limitação de atividades e de movimentação, tipo de calçado utilizado, presença de calos e alinhamento do primeiro raio. Consideramos como resultado satisfatório valor maior ou igual a 70 pontos e insatisfatório os valores obtidos abaixo de 70 pontos. (Tabela 2). A paciente em questão atingiu 62 pontos.

Tabela 2 - Escala de avaliação de hálux valgo - AOFAS

\begin{tabular}{|c|c|c|c|}
\hline DOR (4) pastea) & & Wornetacis WF FP.FD & \\
\hline Nrubora & 46 & 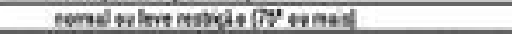 & 19 \\
\hline Leve, scasiohd & $x$ & restigie maderada 3074 ) & 5 \\
\hline 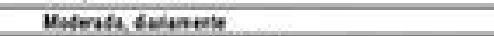 & 29 & gowetessis $2 x$ & $\frac{1}{6}$ \\
\hline Gune quase stopest prestets & 4 & Norinentasis LF FP & \\
\hline runclo a as patal & & sen reftisit & 8 \\
\hline Lintapis 69 effed ad 49 & & grevensticteteles & $\theta$ \\
\hline Nentures & 10 & 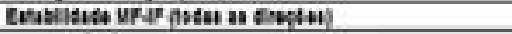 & \\
\hline 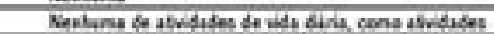 & & etive & 9 \\
\hline 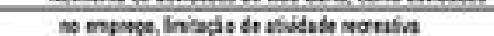 & 7 & 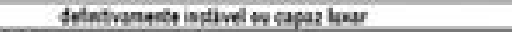 & 8 \\
\hline 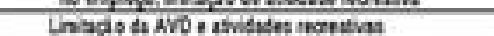 & $\frac{1}{4}$ & 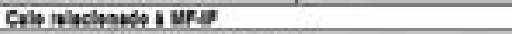 & \\
\hline Crantintacio fas atvitades & 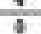 & Un calo ea cale astitonisos & 3 \\
\hline Coleutos & & culetatrailes & 0 \\
\hline 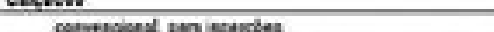 & & 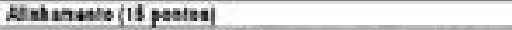 & \\
\hline 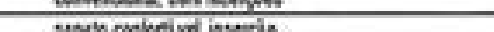 & 16 & ben, Hile ita wiahade & is \\
\hline 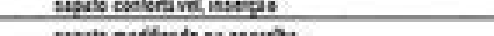 & 5 & 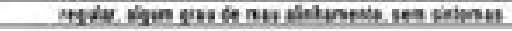 & 8 \\
\hline 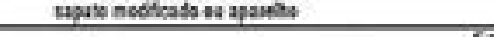 & 0 & 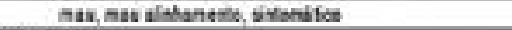 & c \\
\hline
\end{tabular}




\section{DISCUSSÃO}

A cirurgia para correção das deformidades do hálux valgo deve contemplar o conceito de deformidade complexa, que exige uma abordagem visando a correção dos defeitos intrínsecos e análise dos fatores extrínsecos para adequação dos procedimentos e das expectativas do paciente quanto aos resultados possíveis ${ }^{3,4,5,6}$.

A cirurgia proposta foi a técnica de Reverdin-Ishan, osteotomias distal do metatarso e proximal da falange proximal, tenotomia do adutor do hálux e capsotomia lateral, tendo como marco a osteotomia percutânea realizada com analgesia locorregional (divergindo do tratamento cirúrgico clássico, onde há incisão na face lateral da articulação metatarso-falangeana), utilizando-se de brocas que possibilitam cortes laterais tanto distalmente ao metatarso como na falange proximal, guiado por radioscopia. Usualmente não há necessidade de fixação da osteotomia com parafuso, fio de kirshnner ou qualquer outro material de síntese. No pós-operatório, o paciente já é liberado a deambular com apoio de carga total com calçado de solado rígido durante o primeiro mês. A utilização de anticoagulação profilática não é normalmente necessária, exceto se o paciente tiver histórico de eventos tromboembólicos ou fatores de risco (coagulopatias, trombopatias etc.) $)^{2,7}$.

A paciente foi submetida ao tratamento cirúrgico planejado no dia 27.11.2015, no Hospital Santa Izabel. Durante o procedimento, que durou cerca de 30 minutos, não houve intercorrências. (Figuras abaixo)

Durante o posicionamento, as pernas do paciente devem se manter pendentes com apoio no aparelho de radioscopia. Raspas iniciadoras são usadas para descolar a cápsula articular e criar o espaco de trabaIho (Figuras 3 e 4).

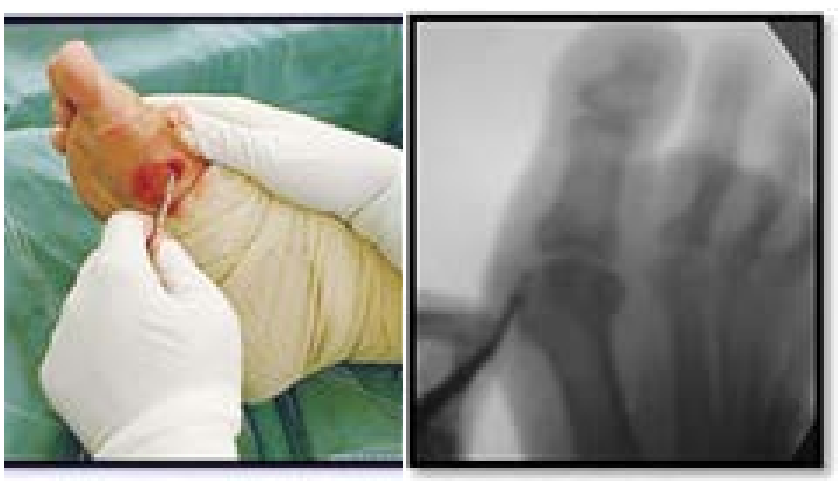

Figuras 3 e 4
Em seguida, faz-se a marcação do local de início de osteotomia e ressecção do bunion (Figuras 5 e 6).

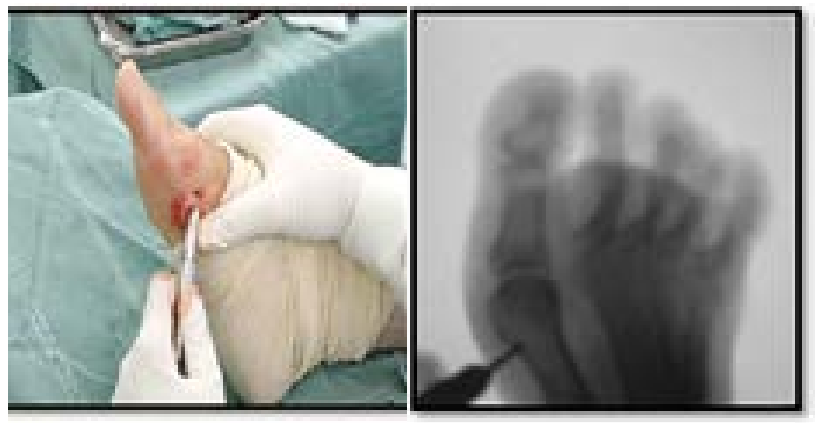

Figuras 5 e 6

As figuras 7 e 8 mostram a realização da osteotomia incompleta e manobra manual de fratura da cortical lateral do osso metatarsal.

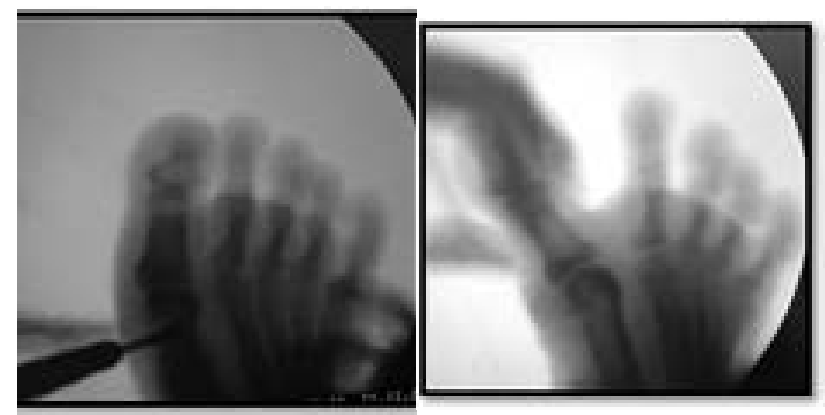

Figuras 7 e 8

Tenotomia e capsulotomia percutânea são realizadas a seguir (Figuras 9 e 10)

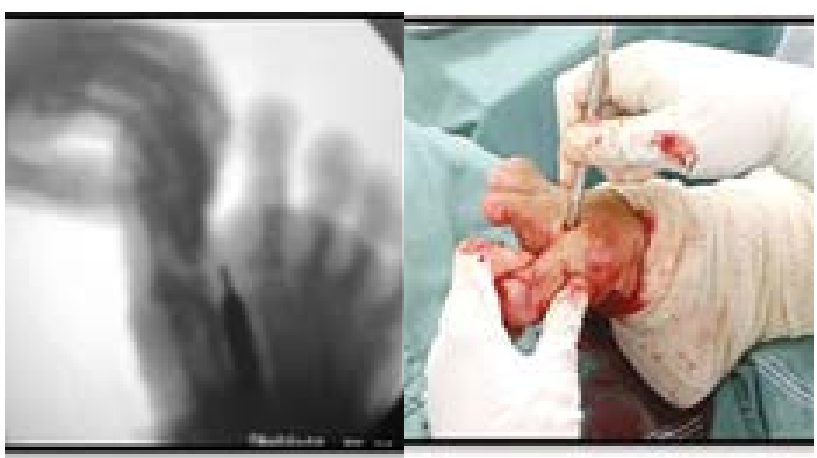

Figuras 9 e 10 


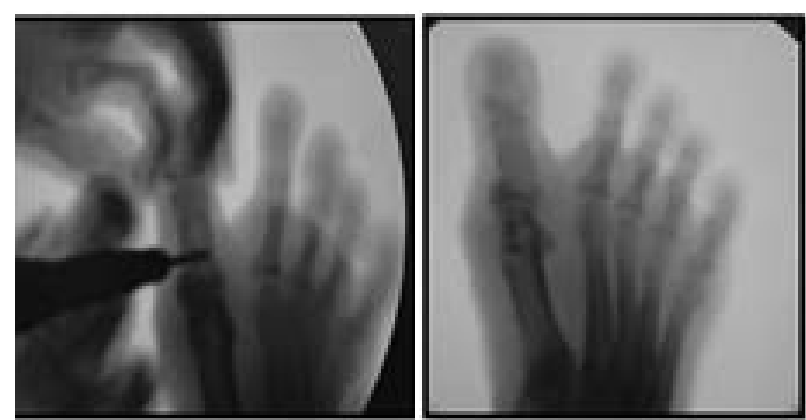

Figuras 11 e 12 - Osteotomia falangeana tipo Akin e controle radioscópico final

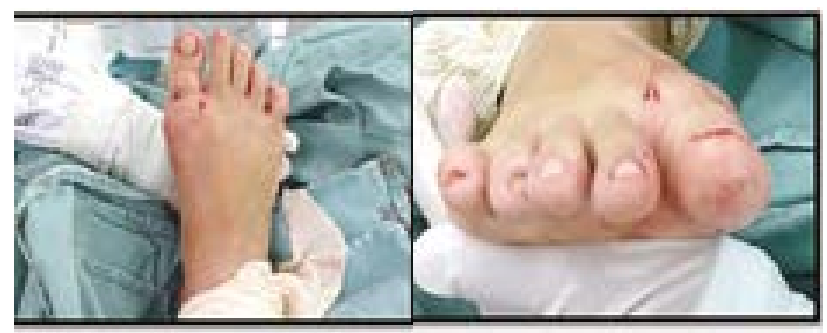

Figuras 11 e 12 - Osteotomia falangeana tipo Akin e controle radioscópico final

A paciente recebeu alta hospitalar no dia seguinte, sendo permitida carga no limite álgico. Foi receitado uso de AINES e analgésicos comuns. Não foi receitado uso de antibiótico ou opioides.

A Figura 15 mostra a paciente com 12 dias de evolução, com miniacessos sem sinais infecciosos, limpos e secos, ainda mostrando edema residual $+/ 4+$, sem alterações na perfusão ou sensibilidade. Paciente não apresentava queixas e já deambulava com carga próxima da total. Os pontos da sutura foram retirados nesse dia.

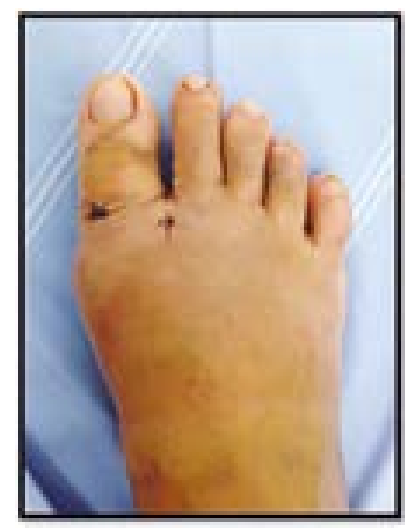

Figura 15
As figuras 16,17 e 18 mostram a paciente no $6^{\circ}$ mês pós-operatório, sem queixas, referindo satisfação com o resultado final, informando desejo de operar o lado contralateral. A escala AOFAS foi novamente aplicada à paciente, atingindo escore 100.
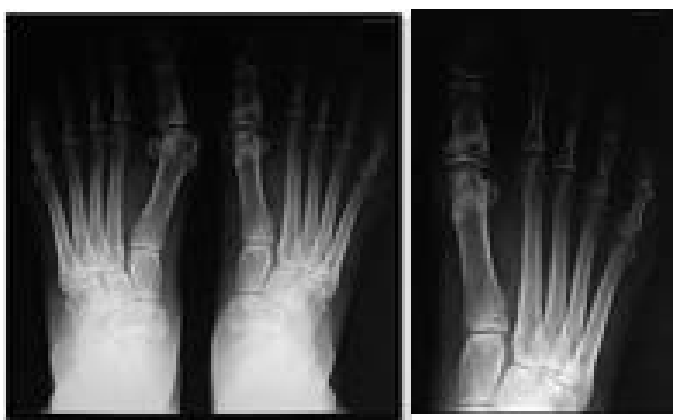

Figuras 16 e 17

\section{Figura 18}

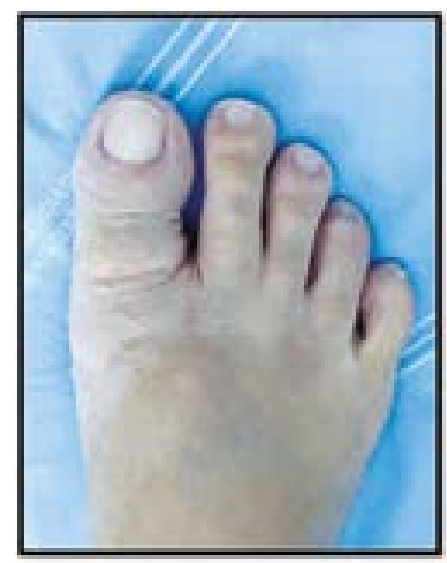

\section{CONCLUSÃO}

A abordagem cirúrgica minimamente invasiva para correção de hálux valgo mostrou resultados clínicos comparáveis às técnicas convencionais, abertas, mesmo quando aplicada para deformidades graves.

O nível de satisfação do paciente com o resultado final pontuou o escore máximo pela escala AOFAS.

Como ponto negativo, tem-se que a técnica necessita de uma longa curva de aprendizado, entretanto o resultado final mostra-se encorajador.

Novos estudos utilizando esta técnica são necessários, a fim de refinar indicações e avaliar a estabilidade a longo termo, bem como a adaptação dos pacientes à utilização de calçados e retorno às atividades cotidianas. 


\section{REFERÊNCIAS}

1. Barros Filho, TEP. Exame físico em ortopedia. São Paulo: Sarvier; 2001.

2. Bauer T. et al. Percutaneous hallux valgus correction using the Reverdin-Isham osteotomy. 2010

3. Campbell WC, Canale ST, Beaty JH. Campbell's operative orthopaedics. 11ed. 2007

4. Cohen, M. Tratado de ortopedia. 1 ed. 2007.

5. Hebert S, Xavier R, Pardini AG Jr., Barros Filho TEP. Ortopedia e traumatologia: princípios e prática. 4. ed. 2009

6. Roger Mann. Surgery of the foot and ankle, 9th Edition. 2014.

7. Trnka HJ,Krenn S, Schuh R. A Minimally invasive hallux valgus surgery: a critical review of the evidence. International Orthopaedics (SICOT) (2013) 37:1731-1735

8. Isham SA. The Reverdin-Isham procedure for the correction of hallux abducto valgus. A distal metatarsal osteotomy procedure. Clin Podiatr Med Surg $1991 ; 8(1): 81-94$.

9. De Prado M, Ripoll PL, Golano P. Cirurgia percutanea del pie. Barcelona: Masson 2003

10. Lara LC, Ferreira C, Prado F, Pires FA, Scardovelli PL. Tratamento cirúrgico do hálux valgo por técnica percutânea. Tobillo y Pie 2014;6(1):vi.

1- Serviço de Ortopedia e Traumatologia do HSI

Endereço para correspondência:

fernandodelmonte@gmail.com 